\title{
A new concept of 3D DCS interface application for industrial production console operators
}

Article in Universal Access in the Information Society · July 2014

DOI: $10.1007 /$ s10209-014-0368-X

CITATIONS

2

6 authors, including:

\section{Ramiro Gonçalves}

Universidade de Trás-os-Montes e Alto Douro 169 PUBLICATIONS 378 CITATIONS

SEE PROFILE

Manuel Pérez Cota

University of Vigo

155 PUBLICATIONS 451 CITATIONS

SEE PROFILE
READS

67
José Luis Bandeira Rodrigues Martins

Universidade de Trás-os-Montes e Alto Douro 69 PUBLICATIONS 179 CITATIONS

SEE PROFILE

\section{Joao Barroso}

Universidade de Trás-os-Montes e Alto Douro 208 PUBLICATIONS 635 CITATIONS

SEE PROFILE

Some of the authors of this publication are also working on these related projects: 


\title{
A new concept of 3D DCS interface application for industrial production console operators
}

\author{
Ramiro Gonçalves · José Martins • Frederico Branco • \\ Miguel R. González Castro • Manuel Pérez Cota · \\ João Barroso
}

Published online: 26 July 2014

(c) Springer-Verlag Berlin Heidelberg 2014

\begin{abstract}
The current operator displays of Distributed Control Systems (DCS) are designed for 2D environments. This limits the full awareness situation of the industrial process, since it is distributed across multiple operator displays and requires the operator to navigate among them. This inspired the idea of creating a single operator display. Through an accurate and systematic literature review and by following the guidelines of design science methodology, it was possible to achieve a set of indicators and artefacts that corresponded to the defined goals. By developing a 2.5D/3D DCS interface application, the authors aimed at creating a way of allowing a full view of the entire manufacturing process, thus increasing the amount and the quality of information that is given to the operator and preventing unnecessary operation navigation between displays.
\end{abstract}

R. Gonçalves $(\bowtie) \cdot$ J. Martins · F. Branco $~ J$. Barroso University of Trás-os-Montes e Alto Douro and INESC TEC (formerly INESC Porto), Quinta de Prados, 5000-801 Vila Real, Portugal

e-mail: ramiro@utad.pt

J. Martins

e-mail: jmartins@utad.pt

F. Branco

e-mail: fbranco@utad.pt

J. Barroso

e-mail: jbarroso@utad.pt

M. R. González Castro

Vigo, Spain

e-mail: miguelrgc@terra.es

M. P. Cota

Escuela de Ingeniería Industrial, DI, University of Vigo,

Rúa Torrecedeira, 86, 36208 Vigo, Spain

e-mail: mpcota@uvigo.es
Keywords User experience $\cdot \mathrm{HCI} \cdot \mathrm{DCS} \cdot 2.5 \mathrm{D} \cdot 3 \mathrm{D}$. Production display operators

\section{Introduction}

Global losses in the continuous process industry have been accounted at the area of $\$ 20$ billion, the equivalent to $5 \%$ of its global annual turnover [1]. Forty percentage of these losses were attributed to mistakes or operation failures, hence implying that the efficiency of the systems operators becomes a critical element of these industries. This demands an increase in the capacity and skills required from the operator, and to the need for providing them with tools and working environments that favour wise decisionmaking.

This industry is characterized by a critical productive process, in which a failure or a shutdown can generate very dangerous situations, both for the environment and for people, outages for several days due to complex breakdowns because of those unplanned failures or shutdowns, or start-ups that can be delayed for several days until the process can be stabilized. This situation requires the use of specific control systems for this type of industry, which are referred to as Distributed Control Systems (DCS) [2], [3], [4]. The DCS manage thousands of analog and digital signals that are controlled through control loops, PID or Artificial Intelligence Systems (Neural Networks or Multivariate Analysis), with some sampling rates ranging between $100 \mathrm{~ms}$ and $2 \mathrm{~s} \mathrm{[5],} \mathrm{[6].} \mathrm{Configuration} \mathrm{is} \mathrm{done}$ through logical blocks, templates or programming libraries designed for these types of industrial processes that must be controlled, making tasks much easier for the teamwork composed of software engineers and productive process engineers. Equally, the DCS has Object Graphics Libraries 
that facilitate the task design of the complex operation displays that should be supervised by the console operator.

The project main goal, inherent to the present paper, is increasing the quantity and quality of information, received by a DCS console, on the status of the industrial process to be controlled. This compels the creation of an innovative DCS operator display that meets the usability principles $[7$, 8]. This improved information allows the operator to acquire knowledge of the current state of the process and thus take thoughtful and reasoned decisions. In addition, it reduces the level of anxiety and increases operator productivity and commitment.

This referred main requirement can be divided into five sub-requirements:

- The operation screen must meet the guidelines or rules applicable;

- The information must be captured by the largest number of senses of the operator;

- The application should run on any operator station;

- The DCS security must be guaranteed from the origin;

- The cost of the tool must be reasonable.

The new operation display must be designed to comply with the spirit of the guidelines and/or recommendations for best practices used in the design of $2 \mathrm{D}$ displays. This will lead to a rapid acceptance and understanding of this new operator interface, which will then be perceived as a natural evolution of 2D screens and not an abrupt break of the current concept of the DCS operator displays.

The software must be designed to run on any computer, regardless of the operating system that is mounted on it. This implies that the DCS interface code and advanced peripherals used by the operator must imperatively be installed on any computer that can use the DCS operator.

The DCS should be completely secure, and it should be avoided that the written code jeopardizes it. The DCS control equipment is used in critical processes, so any software that is related to them must possess a high level of security.

The technical goal is complemented with an economic goal. This requires the use of devices and/or peripherals which are in commercial use. It is important that the cost of the software does not restrict the development of technologies in the industry.

The present paper is divided into five main sections, Sect. 1 being the introduction to the topics approached by the inherent research project. In Sect. 2, a concise and focused characterization of the state of the art is presented. Section 3 entails the description and characterization of the scientific methodology that was followed during the execution of the previously referred research project. In the fourth section, a detailed description of the new 3D DCS interface proposed is presented, and in the fifth section, some brief conclusions and final considerations are made.

\section{Characterization of the state of the art}

As required for all scientific projects, in order for the research team to perform any development and structure any new artefact, a serious and accurate characterization of the state of the art must be made. This will allow for an acknowledgement of the existing knowledge on the research topics [9-13].

\subsection{Operator displays}

An optimal operator interface must provide accurate and complete "situation awareness" in all conditions of the industrial process status (normal, abnormal and emergency). This reduces the number of unscheduled shutdowns, minimizes the variability of the process and improves safety.

Currently, operator displays have a 2D format (see Fig. 1), which hinders access to an accurate knowledge of the state of the process, since it is not feasible to create a single operator display that contains all the information of the industrial process. This requires creating overview displays with the most critical parts of the process and/or forces the operator to navigate through all the displays in the DCS. Both options have advantages and disadvantages, but they do not address the need to view the status of all operating variables, to be monitored in the industrial process.

Operator interfaces are often located in old control rooms not designed according to usability principles. This creates problems of lighting and acoustics in the operator workplace, which must be minimized in operator displays. Moreover, the number of monitors available is insufficient to show all displays in operation, mainly due to the complexity of each display as one can observe in the example shown in Fig. 2.

\subsection{Design guides of operation screens}

Abnormal Situation Management Consortium (ASM) [14] is a consortium of 16 companies and universities, dedicated to the development and investigation of incidents in industrial plants, identifying abnormal operating conditions and proposing solutions. ASM develops guidelines or recommendations about the best practices that can be applied in the design of the operator displays. The purpose of these displays is to optimize the amount and quality of information in the industrial process, which must allow the operator to possess excellent knowledge of the status of the process and thus be able to make the best decisions [15]. 
Fig. 12 D image of a DCS display-Digester $n^{\circ} 8$

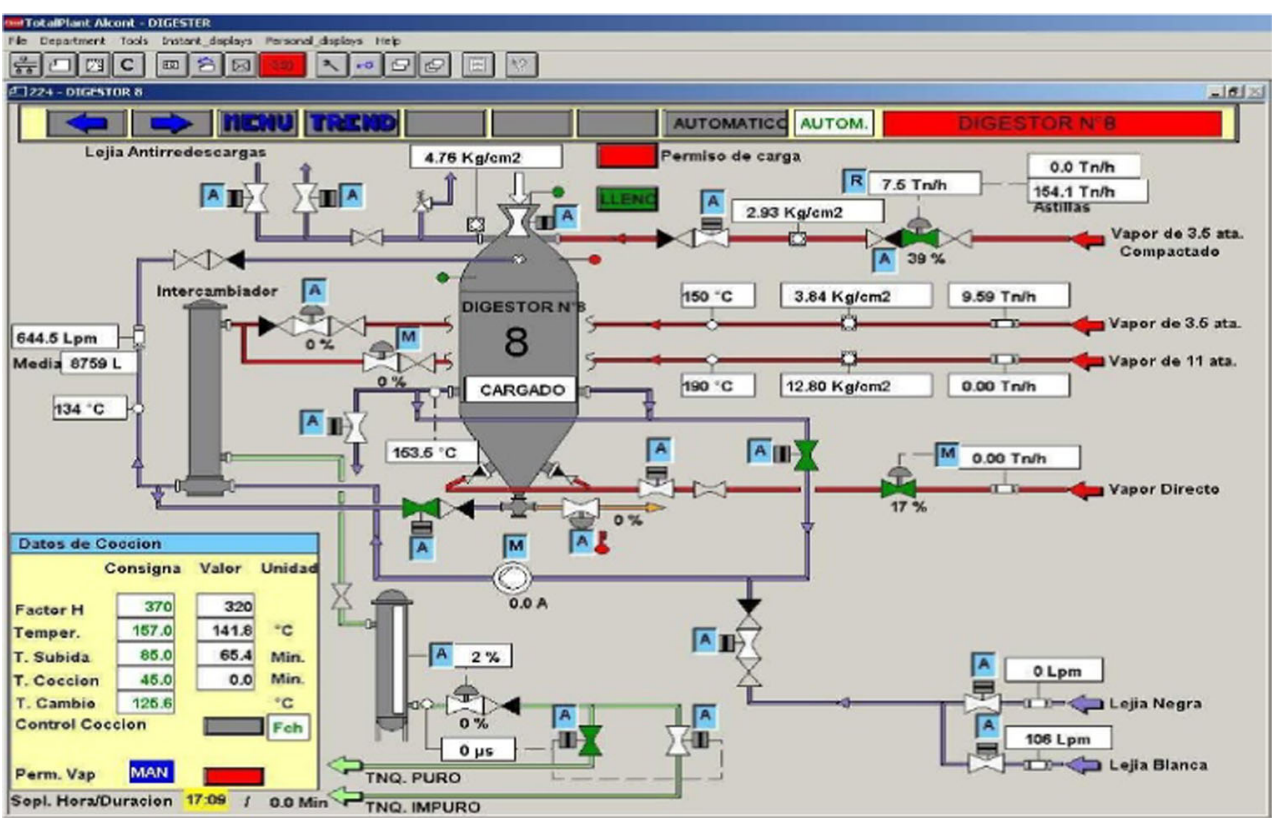

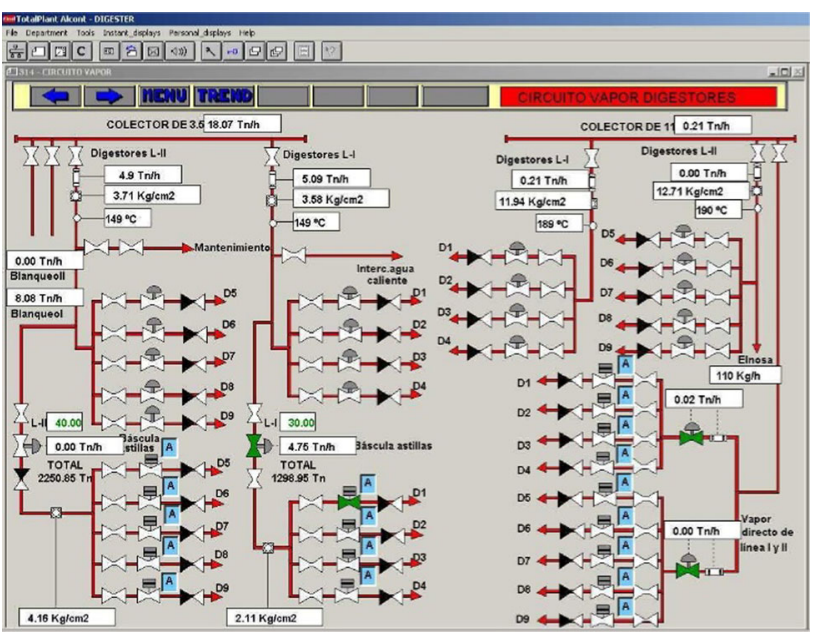

Fig. 2 2D image of a DCS display 2D—steam distribution

These guidelines and recommendations were developed to apply only in 2D displays, but many of them could be extrapolated to design $2.5 \mathrm{D}$ or $3 \mathrm{D}$ displays. A very small summary of these best practices is presented in this paper:

- The graphical symbol of a component must be consistent with its international standard representation;

- Animations should be used to warn of critical situations;

- Displays and symbols must not have excessive detail, to minimize complexity;

- The detail information of an instrument should be visible only if needed;

- The colour coding used in the displays should be composed of a maximum of 7 colours and should not create conflicts with the culture of the operator;
- The red and yellow colours should only be used to report alarms or warnings;

- Background colours should facilitate readability and minimize operator fatigue, with any existing light conditions in the room [16]. Light colours are the most appropriate (grey);

- The colour should be complementary information, and variations of shape, texture or text should also be used;

- The binary status of a component (open/closed, started/ stopped) should be showed with a symbol defined by its edges, while the interior is filled or empty depending on its state;

- The refresh rate of the display data in the DCS should be from 0.5 to $3 \mathrm{~s}$;

- The operator can be informed of a critical or abnormal situation by an audible alarm, whose intensity should be between 15 and $80 \mathrm{~dB}$. The audible alarm may consist of voice messages [17], tone groups and intensity variations, with some repetition frequencies (continuous, burst, etc.);

- Vibration devices can be very useful in noisy environments;

- The operator's viewing angle is $30^{\circ}$, so all critical information should be on the inside of this area of the screen.

\subsection{Actual operator interfaces}

To the authors' knowledge, the majority of the existing DCS operators interface not only with present 2D monitors but also display charts or diagrams containing process in a $2 \mathrm{D}$ format $[18,19]$. This means that if one wishes to have 


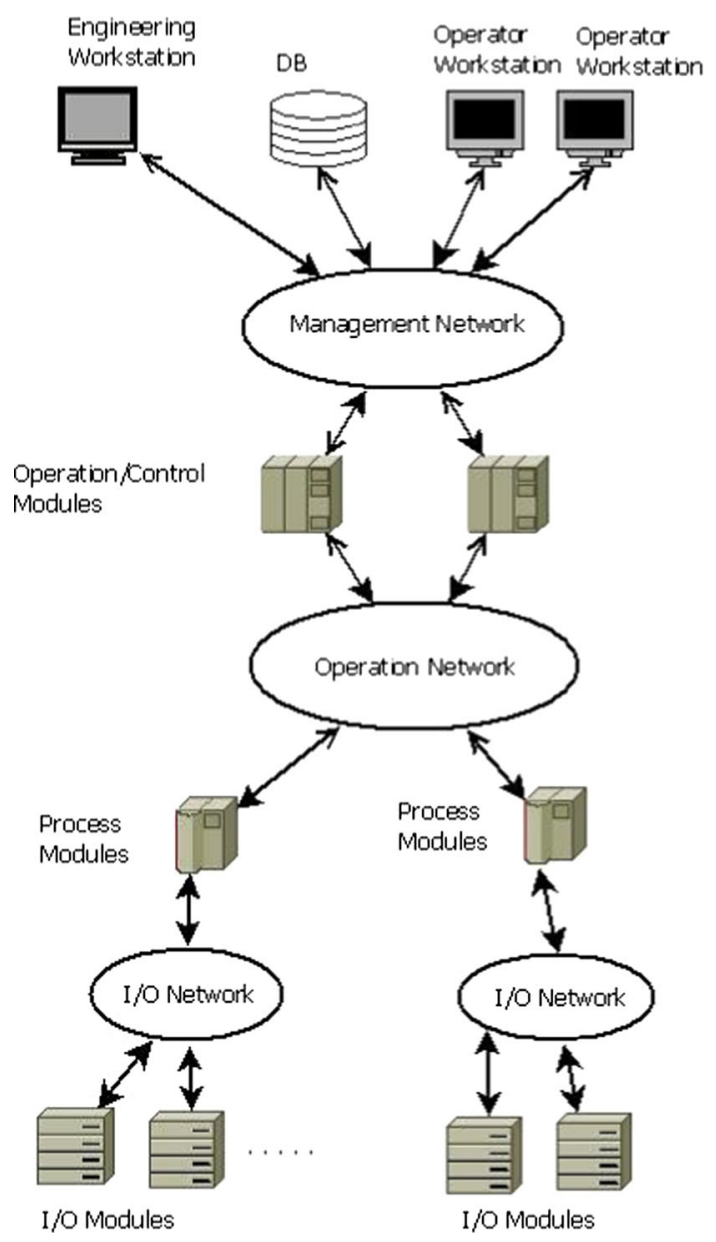

Fig. 3 DCS architecture

different views of the process, it is essential to create new displays. Environmental noise and the existence of multiple workplaces prevent the proper use of voice alarms, since it is difficult to understand their messages and also increases the noise. DCS alarms are indicated on the operator displays, with a change in the colour of the component that generates it, and are also listed in a table sorted by priority and/or areas, which are accessible from different sides of the display. All operator displays show the status of the components using a colour code, while some allow access to programming manuals, device manuals (valve, motor, etc.), programs of logic blocks, wiring diagrams, piping diagrams or links to other management applications (Maximo, SAP, etc.). Moreover, all the components allow the operator to select the operating mode (automatic, manual or remote) or to force their input and/or output values. The typical input interface of DCS is the keyboard and mouse, although in some cases, using a touch screen as advanced device, it supports user-defined configurations, which facilitate access and navigation through the operating displays.
Operators typically manage up to fifty DCS operator displays with several thousand I/O signals associated; in that respect, there may be displays or I/O signals that are not accessed by operators for several working shifts. Sometimes, it happens that these components or displays are not serviced until an alarm or warning sounds (values may be out of range and not in an alarm state).

The DCS, such as those that shown in Fig. 3, have a distributed architecture, because from the engineering station, the configuration is loaded in the centralized and redundant hardware ("control and configuration modules"), while the code is executed in systems ("process modules") which are independent and distributed. It allows that in case of failure of a module, the other modules of the DCS can continue working, thus increasing the reliability, availability and security of these control devices considerably. Moreover, the DCS allows the load of programming blocks without the necessity of shutdown and start-up; this is an obligatory characteristic in control systems that can shut down one or twice a year, though not more often than this.

However, the intensive interaction with the operator is the main characteristic of these systems, as the operator is the one who should take key decisions on the operation of the process that is being controlled. The operator uses those set-points of the control loops to fix the reference values (levels, pressures, intensities, etc.) of the physical variables that the DCS is controlling and, in this way, modifies the state of the process. Besides, operators should decide if the control loops work in automatic, remote or manual mode. In automatic mode, the output values are determined by the operative of the control loop; in the manual mode, the operator forces the output values; in the remote mode, besides the automatic operation, the setpoint is fixed by another control loop. The operator also starts the execution of operation sequences, or he/she operates valves and/or motors that are in manual mode. Equally, the DCS informs him/her about the state of the process, pointing out the existence of warning or critical conditions in the process, as well as shortcomings in the operation of the several actuators or instruments of the process.

\subsection{Vanguard interfaces}

ABB and the Interactive Institute of Umea have developed a prototype of a business management tool [20] which facilitates the monitoring of any Key Process Indicator (KPI), variable or multivariable function to analyse the essential cause of any beneficial or detrimental effect existing in the process. This information, which is displayed in a monitor, is collected from business management systems or from the factory control room. 
This management tool has a monitor with a touch screen, which displays in a $2.5 \mathrm{D}$ environment the production building of the production plant and/or industrial equipment. This screen has touch or multitouch features which facilitate the rotation, displacement, increase or decrease of the scene. This allows new graphical elements and their associated data to appear in the foreground. An overview of the plant shows only high-level KPIs, though if the operator wishes to view a section of the factory, then new KPI or more specific variables emerge, which are specifically related to that area and which were not visible in the upper level before. The data are displayed within a rectangular box that contains the name of the data, its numerical value and a colour bar indicator with variable length.

The application was designed to convert the graphic display in a tool that replaces the traditional projection screen, whiteboard or portfolio. Thus, participants can add comments to the graphical elements in the form of notes, drawings and virtual sticky notes that remain attached to the item and not to a physical position of the display. Similarly, graphics allow access to the display of historical trend data related to them.

The Siemens Comos-Walkinside application [20] facilitates the tasks of management and engineering throughout the life cycle of an industrial plant. This tool maintains all the technical documentation of the industrial plant from the project phase to the operational phase, including the detailed engineering, project execution and design process. Also, the 3D view of the industrial plant equipment facilitates learning and maintenance tasks. This 3D environment is complemented by tabular and textual information in 2D.

ABB software and Siemens Comos-Walkinside were not intended to become a DCS operator interface, not allowing interaction with industrial devices to examine or modify its state. However, these applications show the direction that investigations are heading towards in the human-machine interfaces in industrial management environments, and this suggests that the same trend is transferred to the area of the DCS operator interfaces.

\section{Methodology}

The scientific validity and recognized value of a research project are validated by the scientific methodology in which its execution is based. This section presents a brief description of the present project's inherent methodology.

\subsection{Design science methodology}

The need for improvement in performance and effectiveness represents the main trigger for information systems
(IS) implementations within organizations. In order to fully understand the extent of success achieved by an IS, it is crucial to analyse several items including the following: the IS capabilities, the characteristics of the organization, its human resources, the work routines and the organization's internal development and implementation methodologies [21].

In the IS discipline, the development and enhancement of production and management operations based on the use of information technology are a responsibility that lies with the research teams. This same fact is also true in relation to the development and communication of knowledge concerning management and use of information technologies [22].

In order to achieve the previously mentioned knowledge on DCS interfaces aimed at being used in industrial manufacturing processes, the research team decided to choose a design science (DS) research methodology as the theoretical and supportive research methodology that would help in bringing scientific validity to the project. The use of DS will act as an information feed that will debrief researchers on the interactions among people, technology and organizations that must be managed if the application of an IS is to be considered successful [22].

The DS research methodology is well characterized in the existing IS literature [23, 24, 25]. In a brief manner, one can resume DS as a paradigm directed to develop IT artefacts aimed at solving identified organizational problems [26]. This same paradigm may be applied to the engineering discipline, where several authors have presented contributions extending the application of DS to the development of engineering artefacts [27, 28, 29].

When considering Hevner, March [22], it is possible to identify a set of principles for conducting DS research in the context of IS. These principles were organized in the form of guidelines arguing that a work of research must produce an artefact that addresses a given problem, thus becoming important for the organization's business, and that its utility, quality and efficiency must be rigorously accessed. Finally, the achieved solution should be effectively communicated to the appropriate audiences.

The incorporation of DS in the IS research cycle results in the creation and evaluation of IT artefacts intended to fix identified organizational problems. For the purpose of this paper, the artefact that was developed was a new 3D DCS interface based on design and usability principles. According to the referred methodology, the artefact evaluation can be made by using quantitative and qualitative tests. With this in mind, and by contemplating the organizational context in which the developed artefact will be used, the research team decided to collect qualitative feedback from the organization's human resources in order 


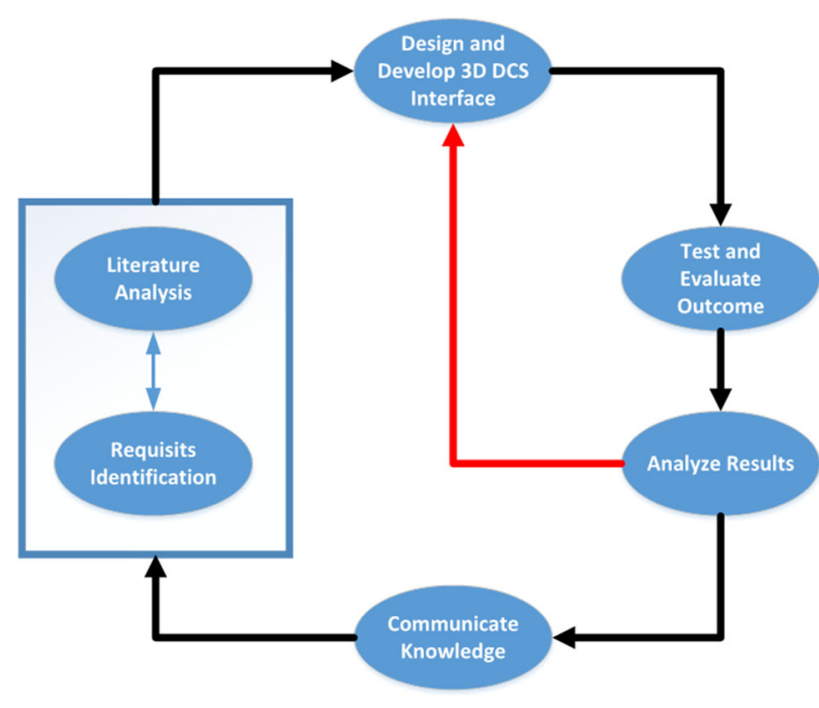

Fig. 4 Project methodology based on DS methodology (adapted from Apedoe, Reynolds et al. 2008)

to perceive the adequacy of the presented solution to the identified problems.

In Fig. 4, one can observe the methodology that supported the research project inherent to the paper. Considering the scope and focus of both design science and the goal behind the project, the methodology considered was based on five stages that would iteratively result in a flow of actions.

The first stage is composed of the following two sets of actions: (1) Requisites Identification-analysis of the existent problems and what might be the possible solutions for fixing it; (2) Literature Analysis_-after identifying the existent problems and the requisites of a possible solution, it is extremely important to analyse the existent scientific literature in order to perceive not only whether the solution to the problem, or to similar problems, has already been discovered, but also to understand what is the best approach for solving the issues identified.

When the research team acknowledged that the scientific know-how, achieved from the literature review, represented a sufficient background to begin designing and developing a possible solution, an evolution to "Designing and Creating a 3D DCS Interface" was made. In this second stage, the most directly related to the engineering discipline, the research team executed works directed at designing and developing a usable and valid 3D DCS Interface that could address all the identified requisites.

As required by DS, in order for a given artefact design and development to be considered an effective solution to the identified problem, it must be tested and validated. In this test and evaluation stage, several tests were performed using real users and real operational situations.
After performing the tests, an analysis of their results was made in order to acknowledge whether the developed 3D DCS interface was a solution to the existent operational problems. This fourth stage, alongside with the second and third, was performed repeatedly until the designed and developed solution fully answered the proposed hypothesis.

When the designed and developed DCS interface was considered to be valid and successful, then the research team undertook the task of communicating the knowledge, inherent to the referred interface, to the organization's appropriate audiences.

\subsection{The research project}

DCS are control technologies used specifically in the continuous process industries, which manage several thousands of analog and digital signals by control loops, PID or Artificial Intelligence Systems (Neural Networks and Multivariate Analysis), with sampling rates that range between $100 \mathrm{~ms}$ and $1 \mathrm{~s}$ [2, 5, 30].

DCS have an architecture that distributes their functions between various modules. Thus, the engineering station is responsible for loading the configuration in the centralized and redundant hardware, while the code runs on systems (processing modules) which are independent and distributed. This allows that, given the failure of one module, the other DCS modules can continue to function, greatly increasing the reliability, availability and security of this control equipment.

The distinguishing feature of this equipment is the intensive interaction between the system and its operator, since this is the person who should make key decisions on the operation of the process being controlled. This is reflected by the inclusion of libraries and graphic objects, which allow the advanced design of operation screens. Operators must decide whether they will control the process in automatic or manual mode, whether they change the parameters of the control loops, or whether to start the sequence of some threads. Similarly, the operator must be alert to possible signs of malfunction in the process, in order to apply appropriate preventive actions to avoid critical interlock.

\subsubsection{Increasing the information presented to the system operator}

The operator of a DCS manages multiple monitors, operating 30-40 displays. In addition, he/she should see all the screens at least once in each 8-h shift, to have at least a mental picture of the entire process during the shift. This tour through all operation displays requires the operator to perform a set of tedious tasks that erode his/her mood and 
concentration. Furthermore, while the operator looks at a display, he/she is unable to observe the rest of the process, which prevents him from holding an exact knowledge of the entire process at any point of his shift. The DCS have audible alarms that do not specify the particular component that active it, so it is imperative to address an operator display to identify which is the component that raises this alarm or warning.

The research project presented in this paper aims at proposing the creation of a new DCS interface, preserving the quality and increasing the amount of information available to the operator. This requires a drastic evolution from a traditional DCS interface, allowing for showing on a single display the entire industrial process operation the operator must control [31].

\subsubsection{Interoperability}

The application created should be interoperable in order to be usable in any industrial environment. In a more detailed level, the main goal behind the current research project is the development of an application that should run on any operator workstation and connect to any DCS.

The connection between the application of the operator interface and the DCS could be made with a proprietary driver, a connection to a TCP/UDP or via OPC. The link to the application with the DCS via a single proprietary driver DCS could be made for those for which the manufacturer provides documentation on how to use the connection driver, though this is often extremely rare. The application connection to the DCS, using a TCP/UDP, would be a feasible option, as all available DCS software allows for a TCP/UDP sockets scheduling; however, this would require creating a specific communication protocol between the application and the DCS, which allows for the exchange of information between the two systems. Similarly, it would also be mandatory to master the programming language inherent to each DCS in order to program the communication protocol between the two systems as well as to create and program a module that would allow reading and writing DCS variables that control the manufacturing process. This connection option would be very expensive and dangerous, because that would require detailed knowledge of the DCS programming languages.

The present paper proposes the use of OPC for supporting the connection between the application and the DCS, since the driver for OPC communication with industrial systems is available for almost all of the existing DCS systems. The main drawback of OPC is the fact that, in order for it to work, an installation on a computer running Microsoft Windows is required, which would be a great disadvantage from the "multiplatform criteria" point of view.

\subsubsection{Security}

OPC is in fact a standard in the market for industrial control systems (PLC and DCS), which allows access to data from these systems by installing special software on computers running Microsoft Windows. This software consists of an OPC server and proprietary driver. The OPC server gives customers access to the data of the industrial system, while the proprietary driver provides bidirectional communication with the industrial system (DCS). The OPC server offers a free and unrestricted security access to any OPC client that is installed on the same computer, which creates a dangerous security breach in the control system.

It is proposed to isolate the computer running the OPC server so that the application of the operator interface has no direct access to it, though through an intermediate software, in order to protect this communication. This said, using Java remote method invocation (RMI) is suggested, to protect this communication with the OPC server. RMI is a Java API designed to locate, call, send and/or distribute Java remote objects with a home security that is embedded in the very core of the API. Moreover, it is intended that the design of the communication architecture with the DCS is divided into two modules, wherein the first module would be installed on the OPC server computer and the second module would be housed in the operator's computer. The first module would be responsible for connecting to the OPC server in order to exchange information with the DCS and to make such information available to the RMI server procedures. At the same time, the second module would own a RMI client for accessing the RMI server procedures in order to read the data collected and transferred to the operator interface software. Similarly, it is proposed to update the information displayed to the operator every 2 or $5 \mathrm{~s}$, while information for the DCS is suggested to be sent immediately, thus avoiding delays that could cause serious damage to the productive process. This option is justified by the fact that information sent to the DCS is critical and a priority. The operator commands to process components (valves, pumps, switches, etc.) should run immediately, thus preventing irreparable damage to these components, unplanned downtime of the production process, environmental mishaps or physical harm to people.

\subsubsection{D visualization}

The 3D visualization is proposed to be implemented through the use of software associated with the 3D hardware (3D graphics card and 3D monitor). This would allow for an update to what is displayed on the operators interface, based on a sequence of 3D images stored in a set of ".jps" files. Hence, this would require the creation of a 
Fig. 5 Oblique view of the industrial process

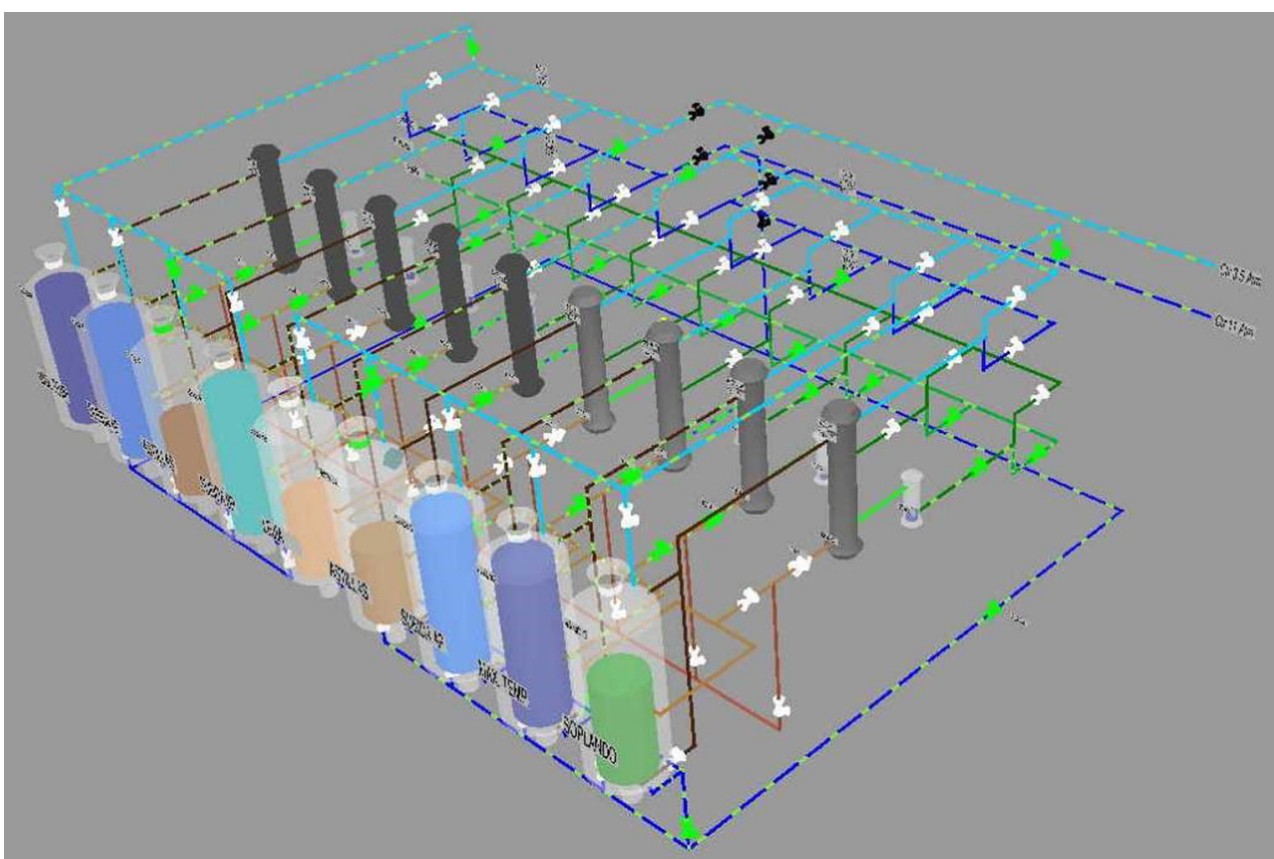

"side-by-side" image (".jps") by the application. Subsequently, this image should be sent to the 3D graphics card image display software, so that the operator could visualize them in 3D. It is recommended sending the images in a sequential manner, as this is a method independent from the OS and from the graphic card drivers. Otherwise, it would be necessary to program the application to support all 3D graphic cards available on the market, which in the authors' point of view would be very costly and timeconsuming, and would transform the proposed application into an unattractive one.

\section{New 2.5D/3D application for DCS interface proposed solution}

In this section, a detailed description of all aspects that support the proposed solution for a new DCS interface application is presented.

\subsection{D application for DCS interface}

An application that shows a DCS operator display in $2.5 \mathrm{D} / 3 \mathrm{D}$ format has been created as the result of the research and the development project inherent to this paper [32]. The application can show a 3D operator display, but can also show operator display in $2.5 \mathrm{D}$ format, on computers that do not have the necessary peripherals to show 3D images. This operator display, presented in Fig. 5, replaces a set of 10 DCS displays with $2 \mathrm{D}$ format. This set of 10 displays is formed by one steam distribution display (Fig. 2) and by the 9 displays of each of the 9 lines of digesters. The display that represents digester number 8 is shown in Fig. 1. Each line of digestion (Fig. 6) is composed of a digester, a heat exchanger, a condensate tank, a recirculation pump, some steam valves, some liquor valves and all associated piping. The steam distribution display shows a set of common facilities for all these lines.

Navigation along the graphic scene is made using a 3D joystick, while the selection of a graphical element is done with the mouse (only 2.5D graphic scene) and/or joystick. Similarly, these peripherals make it easy to spin, pan and zoom the chart of the manufacturing process to achieve visualization of the scene from infinite views (front, side, rear, near, far, etc.).

This operating environment also reports the appearance of an alarm or warning, by issuing a voice message with emotion. The issue of voice messages with emotion facilitates immediate comprehension by the operator of the upsets that are occurring, as emotional intensity indicates the criticality of the alarm and the message indicates exactly which component generated it. The graphic scene also shows, in text, all physical quantities of the process, and the status of all the devices and equipment (valves, motors, switches, etc.).

The application includes a dialog window in 2D format and control information on the process components, which have been selected with the mouse or 3D joystick. This window displays, in textual format, the status of the selected component and allows its control (change of state), by pressing buttons. 
Fig. 6 View of one of the lines of the industrial process

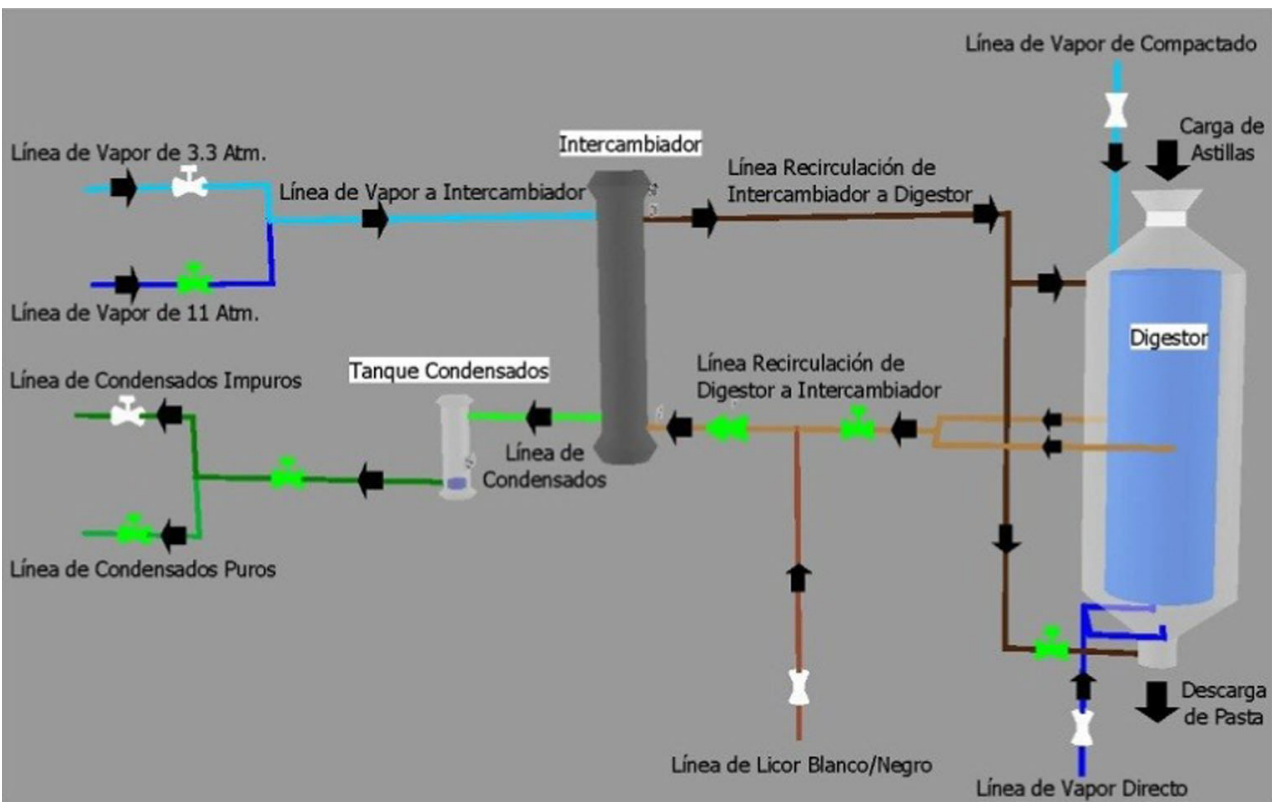

Graphical components must have a geometry format in 3D to be inserted into a graphic scene in three dimensions. However, all standard symbols [33] to identify a component are designed to be used in 2D representations. This requires some new designs of $3 \mathrm{D}$ graphical symbols, to allocate to the process components, so that can be used in a graphic scene to represent a real industrial process. It was decided that these new 3D graphical symbols must be generated by the axial resolution of the graphic representation of the symbol of the 2D component, since this allows them to be recognized or identified in a very simple manner, from any spatial position in which the operator is located. Similarly, the creation of these new symbols from an axial resolution of 2D symbols enables projection onto a 2D plane, which exactly reproduces the original 2D symbol. Occasionally, the axial resolution of the representation of a 2D symbol does not generate a 3D geometric figure, resembling the $2 \mathrm{D}$ symbol. This requires creating a new $3 \mathrm{D}$ symbol, from some geometric transformation of the 2D symbol or by a new reference model for that component.

The digester comprises a geometric revolution of a container with transparent colour, contained inside a cylinder of variable height, which indicates the charge level of the digester. The colour of this cylinder indicates the state in which the digester is (empty, loading chips and/or bleach, temperature rising, baking, puffing, etc.).

The vertical heat exchanger is represented by a vertical cylinder with two flanges at the upper and lower ends. This figure has a solid colour of dark hue and has no operational status that is relevant to know.

The condensate tank is represented by a vertical cylinder with two flanges at the upper and the lower end, containing a cylinder with variable height. The height of this cylinder indicates the level of condensate in the tank, while its colour indicates if it is in a steady state of operation or alarm/warning.

The 3D symbol of a valve is generated by the axial resolution of the normalized $2 \mathrm{D}$ representation of a valve symbol. The 3D symbol comprises the valve body and the handle thereof. The body is represented by a geometrical figure comprised of two circular truncated cones, faced by their smaller radios and joined by a cylinder, whereas the handle resembles a rectangular parallelepiped, which is connected to the body via a cylinder.

The axial resolution of normalized symbol of a 2D pump generates a sphere or a cylinder with a triangular prism inside. None of these geometric figures are easily identifiable with a pump 2D symbol, and the cylindrically shaped figure also has different visual appearance depending on the place where the operator observes it. The normalized symbol of a 2D pump is formed by a circle, within an equilateral triangle, where the vertex indicates the flow direction. Therefore, it was thought to obtain a 3D symbol of the pump, through the axial resolution of said equilateral triangle, but the circular cone obtained is too small to be meaningful. Finally, it was decided that the 3D geometrical figure that represents a pump must be constituted of a set of two circular cones in series, where the two vertices indicate the direction of fluid flowing through the pump.

The red colour is only used to signal an alarm condition, while yellow is used to display a warning status and the violet is identified with a state of uncertainty component. The green and white colours are used to indicate different states of operation in which there is a physical component 
Fig. 7 Partial view of the industrial process

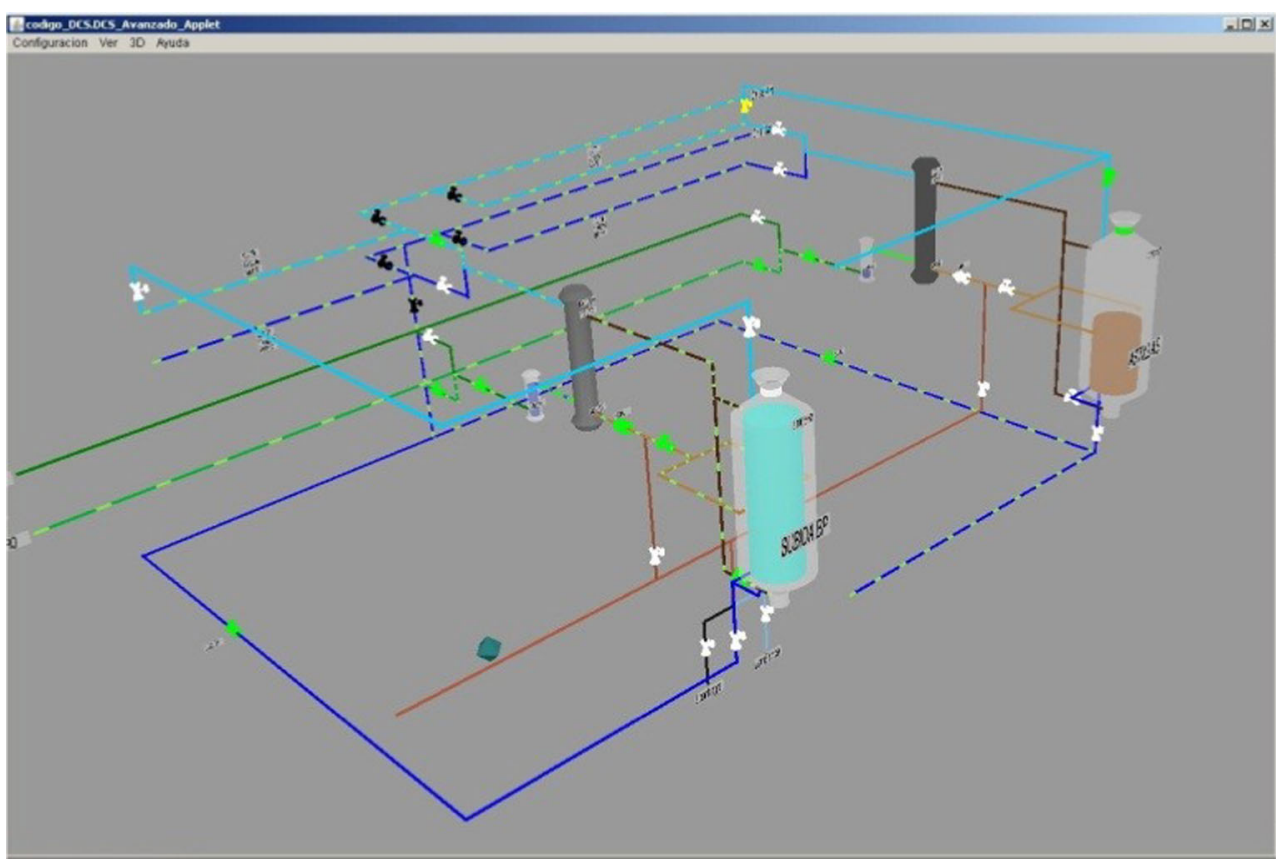

of the industrial process. Green indicates that a pump is running/active or a valve is open. White warns that a pump is stopped/halted or that a valve is closed.

The pipelines have different colours depending on the transporting fluid inside them, while a change in a physical characteristic of a fluid is noticed by a variation in its colour tone. Blue indicates that the pipeline is carrying steam; green denotes that it contains condensates, and brown shows that the pipeline is carrying bleach. The variation of the blue colour tone denotes a change in vapour pressure, for example dark blue indicates that the pipeline transports high-pressure steam and light blue that it contains low-pressure steam. The graphic representation of the pipeline has an animation to symbolize the flow of the carrying liquid. The design of animation imposed the creation of a sequence of bright colour geometries, alternating in a synchronized manner to give the appearance of movement. This sequence is assigned a frequency of $0.1 \mathrm{~s}$ for projecting an optimal illusion of movement.

The graphic scene is contained in a window, with several menus or tabs, which are used to select the different options for modifying the scene. The application allows viewing and/or hiding the individual, pooled or common lines to all digesters (Fig. 7); subsequently, this allows the operator to focus his/her attention exclusively on a limited set of lines. The animation of the pipelines is enabled/ disabled on a menu option, and its activation is highly desirable in simulation tasks or learning the process, as it shows the flow of the various materials involved in the manufacturing process. However, it could be dangerous to have enabled animation pipelines in the routine process control, because the operator could become saturated with the vast amount of visual information given. This could cause the operator to relax while focusing on key aspects of their work of process control and can thus cause malfunction or accident of unpredictable consequences.

Some components or circuit sections should show numerical data or messages to the operator, as a colour code only provides a very limited amount of information. This requires labels to show process information (see Fig. 9) to report the status of the digester or the various physical quantities used to keep the process within its limits. For example, the pump has a label indicating its electric consumption; steam pipelines show a pressure indication that transport fluid; liquor pipelines have an indication of flow and the temperature of the bleach flowing into them; and finally, each process area has a label with an indication of its name or designation.

The moment an alarm or warning arises from a component, the operator cannot concentrate on visualizing the colour change of the component; it is thus advisable to give an audio message to notify the operator that there a circumstance has arisen that requires his/her attention. Therefore, whenever a component gets into alarm or warning, a recorded message is issued, including the name of the component and the critical condition (alarm or warning) that has occurred. Moreover, the menu has a tab that permits the operator to enable or disable such alarm or warning messages. This option is very useful in cases when a lot of alarms or warnings (e.g. when starting the process) occur. This suggests that it would be desirable to disable the broadcast of these messages, in order not to stun the 
Fig. 8 Back view of the industrial process

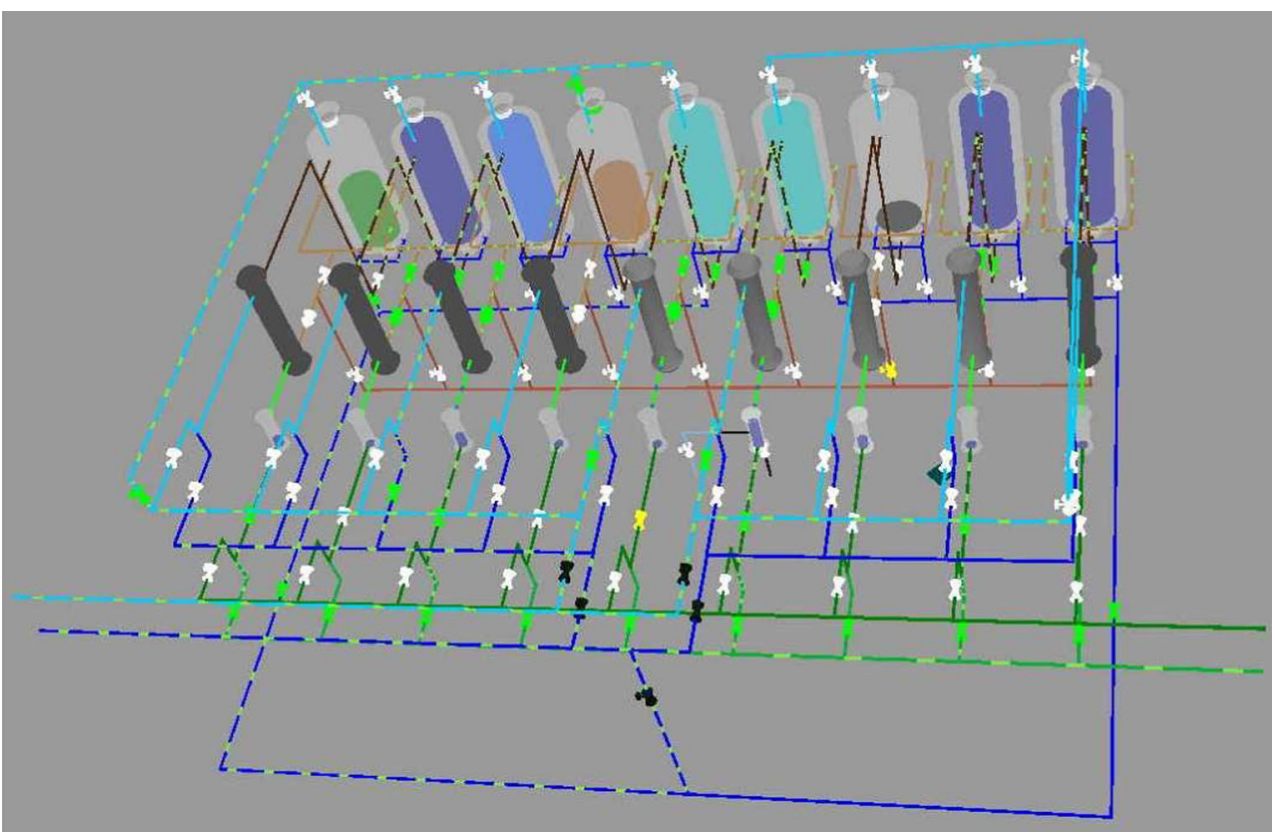

operator with a large amount of sound emissions, which would generate a very noisy environment; this noise could completely distract him/her from their control work of the production process.

The graphic scene, showing the industrial process, has several options that facilitate viewing from other points of view. This implies that the graphic scene can be moved to any position, rotated about any axis or modified in size (zoom). These options allow the operator to navigate inside the process, or to focus on specific areas. This facilitates the visualization of the process from other locations and ensures that no area of the process remains hidden or distorted by any other element that is in front of it. Therefore, the scene can be viewed from the bottom, rear (Fig. 8) oblique (Fig. 5), enlarged, etc. The operator performs these actions using a mouse or a $3 \mathrm{D}$ joystick. The $3 \mathrm{D}$ joystick is the only peripheral device that can be used to interact with the 3D scenes shown, since the graphics card prevents the use of the mouse. Similarly, a menu option or button joystick allows for the reposition of the graphic scene in its original position and thus facilitates this task for the operator.

Components (digesters, valves and pumps) of the industrial process can be selected, so that their information is displayed in a small command window (Fig. 9). This window provides more detailed component information, including its full name and operating state. This window can also be used to change the state of the component, since this option was excluded on the application requirements. Component selection is done with the mouse pointer or by an advanced pointer. The advanced pointer needs to touch or cross a component to make this choice, while the mouse selects the first component located at the same depth coordinate. The advanced pointer is shown on the display with the geometrical figure of an octahedral, and its movement is controlled via a keyboard or 3D joystick. This advanced pointer is only available when viewing $3 \mathrm{D}$ scenes.

\section{$4.23 \mathrm{D}$ visualization}

The stereoscopic 3D view is achieved by obtaining two images of the graphic scene, which correspond to the images that are to be perceived by the left and right eyes of the user. The left eye image is obtained by directly capturing $2.5 \mathrm{D}$ image of the industrial process, though the right eye image is created by a rotation of $7^{\circ}$ of the previous image. The hardware used for $3 \mathrm{D}$ visualization includes a 3D graphics card from Nvidia, a $120 \mathrm{~Hz} 3 \mathrm{D}$ monitor and 3D glasses IR synchronized. The 3D scene cannot permit displaying the mouse pointer, so it is necessary to create a 3D pointer controlled by a 3D joystick. This joystick is the only peripheral that can be used to rotate or move the graphic scene, as well as to move the 3D pointer, which allows for the process components selection. The frame rate of the 3D display is $2 \mathrm{~s}$, which is within the permitted operating range from the ASM.

\subsection{Operator's experience with 3D environments}

The application was validated and tested by DCS console operators of the Digesters Department of the company "ENCE" in Pontevedra (Spain), as well as the Technical Engineering Department of the same company. This 
Fig. 9 Front view of the industrial process, with the commands window

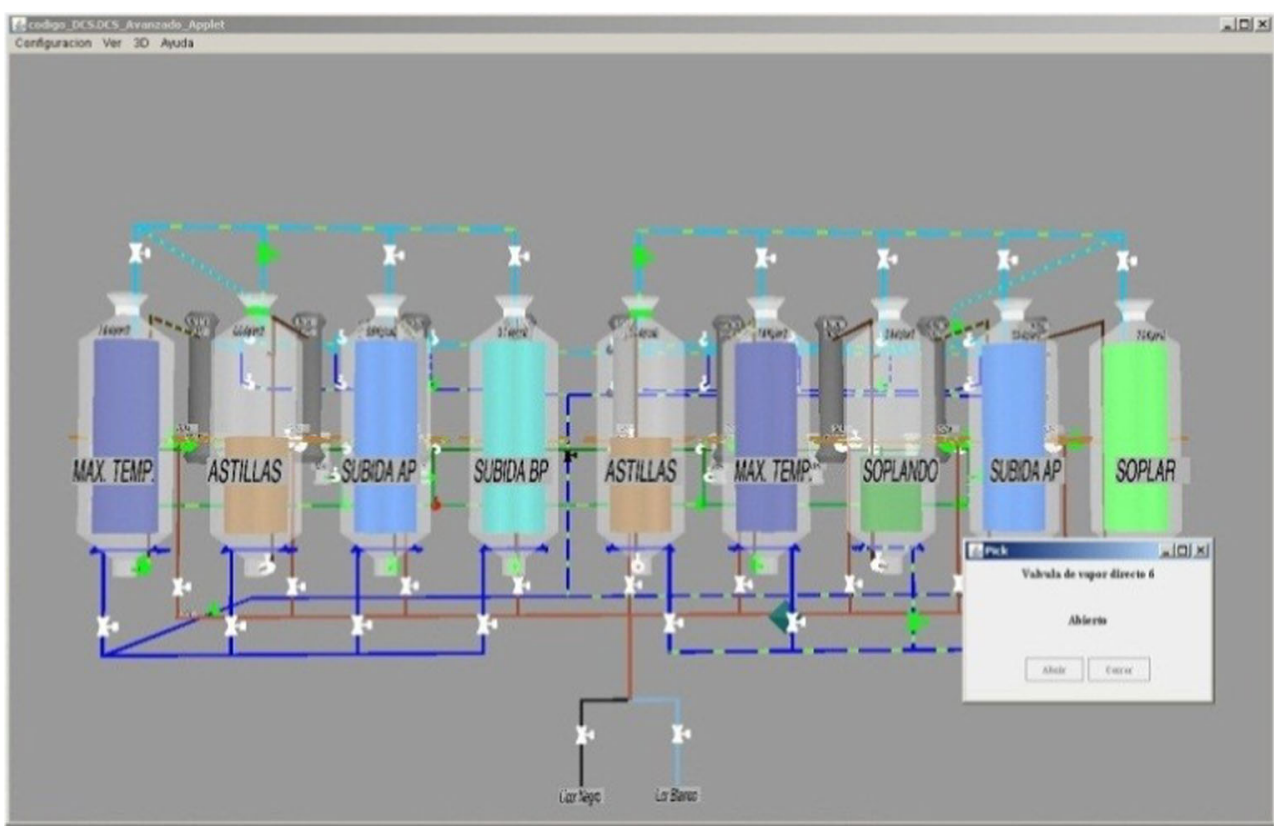

company has installed a DCS "Honeywell-TotalPlant", possessing 2D displays, where each operator is responsible for operating multiple monitors showing various operating displays.

It is observed that the application shows an exceptional quantity and quality of information available to the visual range of operators, as one 3D display shows the same information as 10 old DCS 2D displays. This prevents the operator from having to navigate through all displays (and ignoring what is happening in one display while looking at another). The graphical process has a visual representation that is more natural and intuitive for the user, as it has great similarity to the real process and allows to resemble augmented reality.

Lines or pipelines that are used to connect the components of the process are shown with up to 5 colours, as there is a lot of information at different levels, which could lead to confusion. It avoids the use of extreme tones (dark and light) in the lines and/or pipelines (ASM recommendation), as it is impossible to recognize them in 3D environments, being difficult to distinguish one from another. Also, this creates enormous confusion in the presentation and causes it to be incomprehensible.

The width of the lines is a difficult parameter to use to report the importance of the flow, since the dimensions of the objects are reduced as they are at the bottom of the image. This leads to the inability to distinguish the size of the lines and thereby blurs the information to be transmitted.

The symbols and colours used in the 3D graphic scene are immediately recognized by DCS operators, since the symbols used to represent 3D components are an evolution of $2 \mathrm{D}$ designs proposed by international standards.
This graphic scene transforms hundreds of status signals from the physical devices (valves, pumps, etc.) into visual information based on colours. Previously, this information was displayed in numerical and textual format.

Also, the use of different colours in the representation of 2.5D and 3D figures, showed in the graphic scene, cannot strictly be considered incompatible with usability principles in computer systems. This is because the "risks evaluation" of the workplace of a console operator indicates that this position cannot be attended by people with colour blindness or with some other visual or physical impairment. This evaluation would also be applied to electricians, as an electrician with colour blindness would not be able to perform their work properly, and he/she cannot differentiate all the colours of wires in a cable bundle.

It is observed that the operator requires less mental effort to comprehend the complete state of the industrial process. This allows to assert that the operating display meets key usability requirements, as it is very "user friendly" and very "easy to learn". This is reflected in the way the operator uses all displayed components of the graphic scene.

The flow of information does not always comply with the recommendations of the ASM (from left to right and from bottom to top) because the scene can be rotated in any direction. For example, the back views have inverted process flows recommended, but so are the views up, down, sideways, etc.

The font size decreases as a function of the depth at which text display is located. This leads to the situation where the text has negligible dimensions and is unreadable. 
It is appreciated that operators and users need an adjustment period to the new 3D environment and that they initially are surprised by the original format of the process. Also, some people aged 55 years or older, or visually impaired, are unable to view images in 3D and show that they continue viewing images in 2D, though on the other hand, some young people are extremely receptive to these 3D images. The user's position, the distance to the monitor and the phase angle of each eye images strongly influence the perception of the 3D image by the operator, because the presentation is designed so that it is centred in front of the screen at a distance of $60 \mathrm{~cm}$.

The update rate of the process variables is $2 \mathrm{~s}$, and the speed of 3D rendering images is also $2 \mathrm{~s}$. This implies that the rendering speed for $3 \mathrm{D}$ animations is every $2-4 \mathrm{~s}$, while the velocity in the $2.5 \mathrm{D}$ environment is $0.1 \mathrm{~s}$. However, 3D operators are satisfied with this slowdown, as they believe that there is a great $2.5 \mathrm{D}$ point cloud on screen, which generates a lot of noise in the information in the image.

It is found that 3D images are not suitable as a user interface for some tasks or essential job functions of operation and that those should be shown on 2D displays. In what follows, the features of the DCS interfaces presently not adapted to 3D environments are listed:

- Control charts and/or process variables are more understandable on a $2 \mathrm{D} / 2.5 \mathrm{D}$ display than on $3 \mathrm{D}$;

- Windows command and a process component should be shown on a 2D display, as they have textual, graphical and command buttons;

- The data entry in dialog box (passwords, numbers, etc.) must be programmed in 2D;

- Windows scrolling menus or menus of 2.5D program should not be used in 3D;

- The diagrams in the block of DCS control logic are very complex to follow and understand in $2.5 \mathrm{D} / 3 \mathrm{D}$;

- Help documentation about the process should be displayed in a 2D text format;

- Operator helper applications have to run on auxiliary 2D displays.

The existence of several 3D environments being operated at the same time in a single room tends to create viewing problems to operators in contiguous positions.

\section{Final considerations}

A new concept of a DCS operator interface to be applied to real industrial environments has been introduced, aiming to dramatically increase the amount and quality of process information available to an operator. This new operating environment differs from the current model of reporting by presenting a $2.5 \mathrm{D} / 3 \mathrm{D}$ display perspective instead of the equivalent 10 DCS old 2D displays.
The $2.5 \mathrm{D} / 3 \mathrm{D}$ visual information is more intuitive and natural, permitting the operators to improve their understanding of the real situation of the industrial process. Despite the evident evolution from the existing 2D displays, some of the design techniques used for developing 3D environments are completely unaffordable. This requires adapting these techniques to the new 3D environment, and at the same time trying to retain its essence and implementation costs. Similarly, it has also been found that some features inherent to a DCS interface are hard to transfer to $3 \mathrm{D}$ environments.

The 2.5D/3D symbols used to represent the components constitute an evolution from the existing 2D symbols employed in operator displays, mainly because it is intended that they remain recognizable from any viewing angle. The $2.5 \mathrm{D} / 3 \mathrm{D}$ symbols are created by a $2 \mathrm{D}$ symbol axial resolution. However, some 2D symbols do not allow axial resolution, and a new design has to be created.

Visual information is complemented by sound information and warnings of abnormal or dangerous states in any component of the industrial process. This information is transformed into audio messages with emotion that indicate the warning or alarm to a specific component.

Visual information is also complemented with the use of interaction interfaces, such as a 3D joystick, faster browsing and/or selection in the three-dimensional scene. The use of 3D peripherals allows for rotation, displacement and/or zoom of an industrial process on the screen, thus becoming easier to analyse the information from different angles and locations (front, back, side, oblique, interior, etc.). It is also possible to visualize the inside of the different equipment (digester condensate container, etc.).

Moreover, it is noted that communication between the application and the DCS operator is fully reliable and fast, since it is based on a free and innovative secure model architecture. This model communicates with the DCS through an OPC server, which is protected from any unauthorized access by using Java RMI technology.

Finally, it is concluded that the new DCS operator interface screen, composed of the application and its peripherals, is more usable than the current operator interfaces. This increased usability is complemented by the adaptation for $2.5 \mathrm{D} / 3 \mathrm{D}$ environments of the best guides and design recommendations for 2D screens displays.

\subsection{Future work}

Future research in the area of advanced DCS operator interfaces should steer towards the following main directions: (1) the graphical interface/representation and (2) the associated peripherals.

Innovations in graphics should facilitate visualization of the industrial process with different image formats and 
should also naturally adjust 3D information with the $2 \mathrm{D}$ format information, for the operator. The proposed improvements with regard to visualization include the following:

- The "side-by-side" stereo image displayed in a 3D environment should be generated from a horizontal displacement rather than a graphic scene rotation. This graphical procedure in the world of real cameras is equivalent to using a parallel camera set-up instead of a converging camera "toe-in" set-up. Subsequently, the images obtained by the horizontal displacement should be processed so that they are properly focused on the 3D graphic scene.

- The use of 3D active or passive glasses, to incorporate augmented reality, allows the visualization of 2D graphic superimposed on the industrial process 2.5D/3D. This would facilitate the management of presenting charts, manuals, data states of components and data entry processes.

- The geometric figure that represents a component (valves, pumps, tanks, etc.) in the industrial process should change to a real 3D photography of that component. However, the process will continue to be represented by a flow chart, so that the piping would be displayed by the actual geometry of representation.

- The scene graph should display a 3D real image of the industrial process, which would be obtained by real 3D photography made in the plant. The status should indicate components by changing their colour or by some other innovative format.

Peripherals attached to the application should facilitate the operator performing complex and tedious tasks to get them to focus on tasks that bring added value to process management and control. In addition, these devices should improve the way they communicate to the operator the instantaneous state of the industrial process, though without saturating the senses with superfluous information. The following are possible areas for the improvement of these advanced peripherals:

- The operator should wear wireless headphones to listen to alarms or notices issued in the DCS format voice messages with emotion [17]. The use of wireless headphones can reduce the ambient noise level of the room and prevent interference between different positions alarms. This solution would also allow operators to shift from their post without missing on possible alarms.

- Alarms, especially critical ones, should inform the screen console operator and floor manager by issuing a "sonic pulse", to make them aware of the criticality of the situation. The sonic pulse is a subsonic frequency sound wave at high intensity, which is emitted by a vibration device located on the wireless headset or the receiver arm.

- The use of haptic feedback should be used in virtual consoles with multiple dashboards [34]. This would allow the introduction of data (numbers and text), manipulation of sliders and button pushing.

- Using active haptics would facilitate intuitive reception of information in $2.5 \mathrm{D} / 3 \mathrm{D}$ environments. This would allow for touching a line (power line). These assets provide haptic feedback on the physical quantities (temperature, pressure or voltage) of the fluid (electricity). Fluid pressure from a pipe or a line voltage could be communicated via a vibration device [148], where the vibration intensity would be proportional to the measured physical quantity.

- The graphic scene browsing should be performed by a navigation device, which would take into account the movement of the pupil and/or the head [35] of the operator. This would free the operator from using his/ her hands and improve the speed of querying the status of industrial process components.

\section{References}

1. Breibvold, H., et al.: El operario eficaz. Revista ABB 4(10), 6-11 (2010)

2. Cota, M.P., Castro, M.R.G.: DCS 3D Operators in Industrial Environments: New HCI Paradigm for the Industry. In: Shumaker, R. (ed.) Virtual, augmented and mixed reality. systems and applications, pp. 271-280. Springer, Heidelberg (2013)

3. Heck, B., Wills, L., Vachtsevanos, G.: Software technology for implementing reusable, distributed control systems. In: Valavanis, K. (ed.) Applications of intelligent control to engineering systems, pp. 267-293. Springer, Netherlands (2009)

4. Stouffer, K.A., J.A. Falco, and K.A. Scarfone, SP 800-82. Guide to Industrial Control Systems (ICS) security: supervisory control and data acquisition (SCADA) systems, distributed control systems (DCS), and other control system configurations such as programmable logic controllers (PLC), National Institute of Standards \& Technology (2011)

5. Nelson, B. and T. Stauffer, PLCs and DCSs convergence. control engineering, 2008. 55(5)

6. Holley, D.W.: Understanding and using OPC for maintenance and reliability applications. Comput Control Eng 15(1), 28-31 (2004)

7. Pittarello, F.: Accessing information through multimodal 3D environments: towards universal access. Univ Access Inf Soc 2(2), 189-204 (2003)

8. Calleros, J.M.G., García, J.G., Vanderdonckt, J.: Advance human-machine interface automatic evaluation. Univ Access Inf Soc 12(4), 387-401 (2013)

9. Cupec, R., Aleksi, I., Schmidt, G.: Step sequence planning for a biped robot by means of a cylindrical shape model and a highresolution 2.5D map. Robot Auton Syst 59(2), 84-100 (2011)

10. Suau, X., Ruiz-Hidalgo, J., Casas, J.R.: Real-time head and hand tracking based on 2.5D Data. Multim IEEE Trans 14(3), 575-585 (2012) 
11. Hoogen, W.V.D., et al.: Visualizing the third dimension in virtual training environments for neurologically impaired persons: beneficial or disruptive? J Neuro Eng Rehabil 9(1), 73 (2012)

12. Vyatkin, V.: IEC 61499 as enabler of distributed and intelligent automation: state-of-the-art review. Ind Infor IEEE Trans 7(4), 768-781 (2011)

13. Leitão, P.: Agent-based distributed manufacturing control: a state-of-the-art survey. Eng Appl Artif Intell 22(7), 979-991 (2009)

14. ASM. ASM consortium. 2012; Available from: http://www.asm consortium.net/ Cited 2 Feb 2013

15. Errington, J., et al.: Establishing human performance improvements and economic benefit for a human-centered operator interface: an industrial evaluation. Proc Hum Fact Ergon Soc Ann Meet 49(23), 2036-2040 (2005)

16. Bullemer, P., Reising, D., Laberge, J.: Gray backgrounds for DCS operating displays. Control Engineering (February 2011), 2011

17. Baldassarri, S., Cerezo, E., and Anaya D.: Interacción emocional con actores virtuales a través de lenguaje natural. In: Macías, J., Granollers, A., Latorre, P., (Eds.) Interacción 2007-VIII Congreso Internacional de Interacción Person-Ordenador. (pp. 343-352) Spain (2007)

18. Cota, M.P., Castro, M.R.G.: Interfaz Avanzada de Oprador de DCS, in $6^{\text {a }}$ Confêrencia Ibérica de Sistemas e Tecnologias de Informacao2011: Chaves, Portugal. pp. 37-41

19. Panigrahy, R.K., et al.: Steel plant production line automation strategy using advanced distributed control and convergent technology. Int J Autom Control 6(2), 157-173 (2012)

20. Olausson, M., Larsson, M.: Colaborando en un nueva dimensión. Revista ABB 2(12), 6-11 (2012)

21. Silver, M.S., Markus, M.L., Beath, C.M.: The information technology interaction model: a foundation for the MBA core course. MIS Q 19(3), 361-390 (1995)

22. Hevner, A.R., et al.: Design science in information systems research. MIS Q 28(1), 75-105 (2004)

23. Benbasat, I., Zmud, R.: Empirical research in information systems: the practice of relevance. MIS Q 23(1), 3-16 (1999)
24. Denning, P.J.: A new social contract for research. Commun ACM 40(2), 132-134 (1997)

25. Baskerville, R., Pries-Heje, J., Venable, J.: Soft design science methodology, In Proceedings of the 4th international conference on design science research in information systems and technology 2009, ACM: Philadelphia, Pennsylvania. p. 1-11

26. Peffers, K.E.N., et al.: A design science research methodology for information systems research. J Manag Infor Sys 24(3), 45-77 (2007)

27. Eekels, J., Roozenburg, N.F.M.: A methodological comparison of the structures of scientific research and engineering design: their similarities and differences. Des Stud 12(4), 197-203 (1991)

28. McPhee, K.: Design theory and software design, Department of Computing Science. University of Alberta, Alberta (1996)

29. Archer, L.B.: Systematic method for designers. In: Cross, N. (ed.) Developments in design methodology, pp. 57-82. Wiley, London (1984)

30. Herb, S.: Hybrid (PAC) systems: pathway to productivity. Assembly Autom 28(1), 10-17 (2008)

31. Mieczakowski, A., Langdon, P., Clarkson, P.J.: Investigating designers' and users' cognitive representations of products to assist inclusive interaction design. Univ Access Inf Soc 12(3), 279-296 (2013)

32. Cota, M.P., Castro, M.R.G.: Usability in a new DCS interface, in universal access. In: Stephanidis, C., Antona, M. (eds.) Humancomputer interaction. Design methods, tools, and interaction techniques for einclusion, pp. 87-96. Springer, Heidelberg (2013)

33. ISO. ISO-International Organization for Standardization. 2013 [cited 2013 5th of March]; Available from: http://www.iso.org/ iso/home.html

34. Borst, C.W., Volz, R.A.: Evaluation of a haptic mixed reality system for interactions with a virtual control panel. Presence Teleop Virtual Environ 14(6), 677-696 (2005)

35. Pallejà, T., et al., Ratón Virtual Relativo Controlado con los Movimientos de la Cabeza in IX Congreso Internacional Interacción2008: Albacete, Spain. p. 305-314 